\title{
Abundancia y biodiversidad de los macroinvertebrados del río Oja (La Rioja, España)
}

\author{
María Valladolid ${ }^{1, *}$, Juan José Martínez-Bastida ${ }^{2}$, Mercedes Araúzo ${ }^{2}$ y Carmen Gutiérrez ${ }^{3}$ \\ 1 Dep. Biodiversidad y Biología Evolutiva, Museo Nacional de Ciencias Naturales (CSIC), c/ José Gutiérrez \\ Abascal, 2. 28006 Madrid. \\ 2 Dep. Contaminación Ambiental Centro de Ciencias Medioambientales (CSIC), c/ Serrano 115 dpdo., 28006 \\ Madrid. E-mail: J.J. Martínez: Martinez.Bastida@ccma.csic.es, M. Araúzo: mercedes@ccma.csic.es \\ 3 Departamento de Agroecología. Centro de Ciencias Medioambientales (CSIC). Serrano 115 dpdo. 28006 \\ Madrid. E-mail: carmen.g@ccma.csic.es
}

Autor responsable de la correspondencia: marval@mncn.csic.es

\section{RESUMEN}

\begin{abstract}
Abundancia y biodiversidad de los macroinvertebrados del río Oja (La Rioja, España)
En mayo de 2003 se realizó un estudio sobre la fauna de invertebrados del río Oja (La Rioja, España), seleccionándose cinco puntos a lo largo de su cauce. Se observa una disminución de la diversidad desde los tramos más altos hasta los más bajos, así como un aumento de la abundancia total, representada por unas pocas especies. En los tramos altos dominan Tricópteros, Plecópteros y Efemerópteros, mientras que en los tramos medios-bajos los Tricópteros prácticamente desaparecen, siendo los Dípteros, Crustáceos y ciertas especies de Efemerópteros los principales representantes.
\end{abstract}

Palabras clave: Macroinvertebrados, diversidad, río Oja.

\begin{abstract}
Abundance and biodiversity of macroinvertebrates of the Oja River (La Rioja, España)

During May 2003 a study on the aquatic macroinvertebrate fauna from the Oja River (La Rioja, Spain) was carried out in five selected points along the river. A decrease in diversity from upstream to downstream stretches is observed, as well as an increase in the total abundance, represented by a few species. In the upstream stretches, the dominant groups were Trichoptera, Plecoptera, and Ephemeroptera, while in the middle and downstream stretches, Trichoptera almost disappear, being Diptera, Crustacea and certain Ephemeroptera the most abundant taxa.
\end{abstract}

Keywords: Macroinvertebrates, diversity, Oja River.

\section{INTRODUCCIÓN}

La Rioja y su entomofauna acuática ha sido objeto de estudio desde principios del siglo pasado, debido sobre todo a los trabajos de Navás (p.e. Navás, 1914, 1917a y b), en zonas muy concretas de la región, como Ortigosa, Valvanera y la Sierra de Cameros. Más recientemente, García de Jalón (1982), en su zoogeografía de tricópteros, publica una relación de especies encontradas en los ríos Iregua y Nájera. Por otra parte, hemos observa- do una escasez de literatura referida al río Oja en sí, encontrando por el momento únicamente un artículo sobre los coleópteros acuáticos del Sistema Ibérico en la que aparece, entre otros, este río (Valladares et al., 2000).

El presente trabajo quiere ser una contribución al conocimiento de la fauna acuática de invertebrados de La Rioja, poco estudiada en muchos aspectos y posiblemente vulnerable, debido al gran desarrollo agrícola de la zona y con la explotación de los recursos hídricos como su prin- 
Tabla 1. Localización de los puntos de muestreo y características físico-químicas de las aguas del río Oja. Location of sampling sites and physico-chemical characteristics of the Oja River waters.

\begin{tabular}{cccccccccc}
\hline Punto & UTM & Alt. $\mathbf{~}$ & $\mathbf{T}^{a}$ agua ${ }^{\circ} \mathbf{C}$ & Cond $\boldsymbol{\mu}$ /cm & Sal. g/l & $\mathbf{O}_{\mathbf{2}} \mathbf{~ m g / l}$ & $\mathbf{p H}$ & DQO $\mathbf{~ m g / l}$ & $\mathbf{S S ~ s s ~} \mathbf{~ m g / l}$ \\
\hline R-1 & 30T 496378 4675906 & 944 & 7.02 & 12 & 0.01 & 15.57 & 5.63 & 1.00 & 0.80 \\
R-2 & 30T 499284 4687396 & 719 & 11.88 & 109 & 0.07 & 13.50 & 7.22 & 1.60 & 0.40 \\
R-3 & 30T 502037 4693135 & 711 & 11.51 & 135 & 0.11 & 13.86 & 7.44 & 2.20 & 0.53 \\
R-4 & 30T 503531 4702049 & 616 & 16.81 & 210 & 0.12 & 12.46 & 8.01 & 4.20 & 2.33 \\
R-5 & 30T 507001 4710589 & 569 & 15.28 & 216 & 0.15 & 10.28 & 7.54 & 12.20 & 5.20 \\
\hline
\end{tabular}

cipal amenaza. En esta publicación presentamos la lista de las especies de invertebrados acuáticos que hemos encontrado, su abundancia y diversidad, completando la información sobre estas comunidades y su relación con la calidad del agua en una segunda publicación en este mismo número de la revista (Martínez Bastida et al., 2006).

\section{ÁREA DE ESTUDIO}

El estudio se llevó a cabo en el río Oja (La Rioja, España) (Fig. 1). Este río nace en la Sierra de la Demanda (1880 msnm) y desemboca en el río Tirón, poco antes de su desembocadura en el Ebro, estando su recorrido $(47 \mathrm{~km})$ circunscrito íntegramente a la Rioja Alta. De régimen pluvionival, este río se caracteriza por grandes variaciones de caudal entre distintas épocas del año, debido por una parte a la fuerte pendiente de su cauce, que hace que la escorrentía sea importante, y por otra, a la fuerte utilización del acuífero aluvial para la agricultura intensiva, con importantes extracciones de agua en los momentos de menor caudal (verano).

Se han seleccionado cinco puntos de muestreo a lo largo del río: dos de cabecera, R-1, por encima de Posadas, un poco antes de la unión del Oja con el arroyo Ortigal y R-2, en Ezcaray, por debajo de los efluentes de la depuradora. Dos puntos en el tramo medio, R-3, Santurde, a la entrada del valle aluvial, y R-4, Santo Domingo de la Calzada, en plena zona de regadío, y un último punto en el tramo inferior, R-5, Casalarreina, poco antes de su unión con el río Tirón. El tramo medio (R-3 y R-4) aparece seco durante el verano, recuperando su caudal con la llegada de las lluvias.

\section{MATERIAL Y MÉTODOS}

Para la caracterización físico-química del agua se tomaron por una parte muestras para su análisis en el laboratorio, y por otra se midieron in situ una serie de variables $\left(T^{a}, \mathrm{O}_{2}\right.$ disuelto, $\mathrm{pH}$, conductividad, salinidad) mediante una sonda multiparaparamétrica YSI 556.

Para el estudio de los invertebrados se tomaron un número variable de muestras en cada punto (mínimo 6, máximo 12), dependiendo de la anchura del río. Para ello se utilizó un dispositivo tipo surber $30 \times 30 \mathrm{~cm}$, con una manga de nytal de $250 \mu \mathrm{m}$ de luz de malla. Al mismo tiempo, se colocaron aguas arriba redes de deriva de $30 \times 30$ $\mathrm{cm}, 250 \mu \mathrm{m}$ de luz de malla, durante 30 minutos. Las muestras se fijaron con formol al $4 \%$ tamponado con $\mathrm{ClNa}$ al $0.9 \%$. En el laboratorio los organismos se separaron con una lupa binocular y se determinaron con la máxima resolución taxonómica posible, conservándose en alcohol de $70^{\circ}$. Para la determinación se utilizaron distintos tipos de claves (p.e. Bertrand, 1954; Puig, 1984, Vieira, 2000; Tachet et al., 2003), así como descripciones de ciertas especies.

Se calculó la abundancia de cada grupo taxonómico y la abundancia total en cada punto de muestreo, expresada en individuos $/ \mathrm{m}^{2}$. La diversidad se calculó mediante el índice de ShannonWeaver $\left(H^{\prime}\right)$ y el de Pielou $\left(J^{\prime}\right)$.

\section{RESULTADOS}

En la Tabla 1 aparecen reflejados algunos resultados de las características físico-químicas del agua. Son aguas limpias, de buena calidad, con 


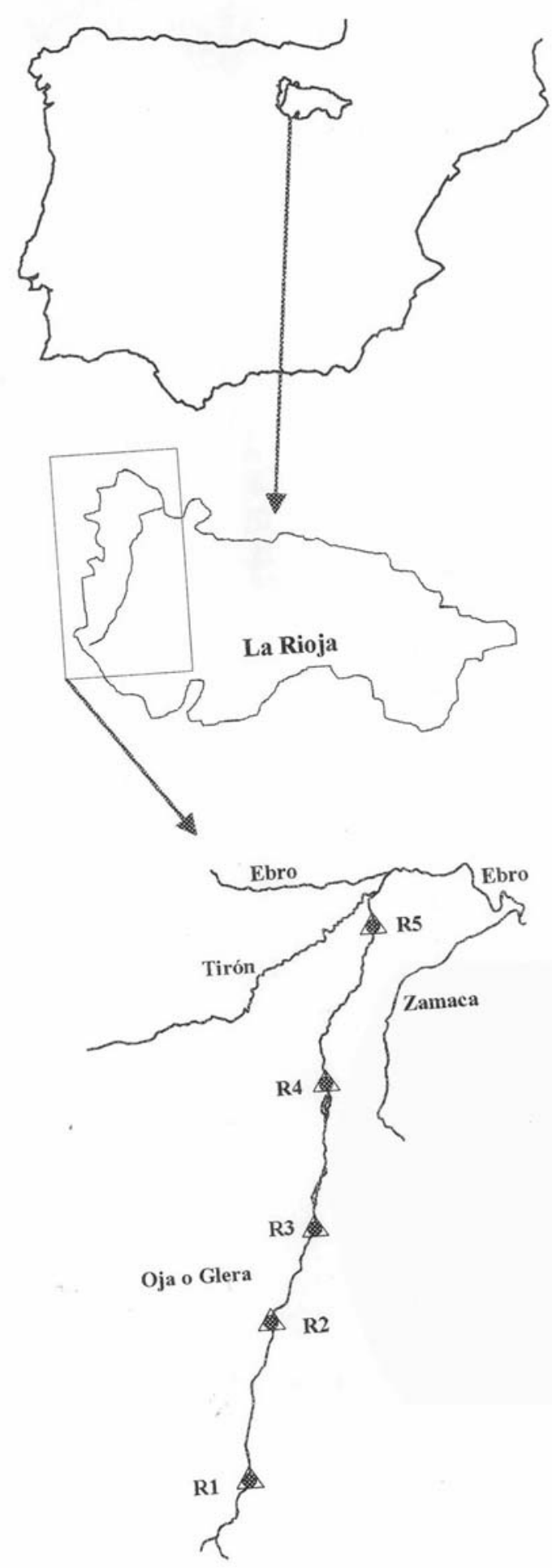

Figura 1. Localización del río Oja (La Rioja, España) y de las estaciones de muestreo. Location of the Oja River (La Rioja, Spain) and the sampling stations. baja conductividad y buena oxigenación, bastante ácidas en la cabecera. La relación entre variables ambientales y el macrobentos se presenta con detalle en Martínez Bastida et al. (2006).

En cuanto a los invertebrados, la Tabla 2 recoge las abundancias de las especies encontradas. Los datos más importantes son la presencia de una posible nueva especie del género Threm$m a$ (Trichoptera: Uenoidae), (pendiente de confirmar, debido a que solo hay un ejemplar), que apareció en el punto R-1 (cabecera), y la relación de nuevas citas de tricópteros, plecópteros y coleópteros para La Rioja, señalados con un asterisco (Tabla 2). Entre éstas destacan especies citadas como endémicas de Pirineos, como p.e. Isoperla moselyi (Plecoptera: Perlodidae) (Despax, 1936; Aubert, 1963; Puig, 1980) u otras encontradas hasta el momento únicamente en el sur peninsular, como Hydropsyche fontinalis (ZamoraMuñoz et al., 2002).

En los tramos altos (R-1 y R-2) se observa la mayor riqueza de tricópteros, plecópteros, efemerópteros y coleópteros, siendo menor en R-2 debido probablemente al vertido de la depuradora. En ambos puntos hay un total de 41 taxa: tricópteros (7 familias), plecópteros (6 familias), efemerópteros (5 familias), coleópteros (3 familias), dípteros (8 familias), colémbolos (1 familia), crustáceos (5 órdenes), moluscos (1 familia), tricládidos (1 familia), hirudíneos (2 familias) y oligoquetos (3 familias). En el tramo medio (R-3 y R-4) encontramos 30 taxa: tricópteros (1 familia), plecópteros (4 familias), efemerópteros (5 familias), coleópteros (3 familias), dípteros (12 familias), colémbolos (1 familia), crustáceos (4 órdenes), hirudíneos (1 familia) y oligoquetos (3 familias). Por último, en el tramo bajo (R-5) únicamente aparecen 26 taxa: tricópteros (1 familia), plecópteros (1 familia), efemerópteros (4 familias), coleópteros (1 familia), dípteros (5 familias), colémbolos (1 familia), hemípteros (1 familia), crustáceos (4 órdenes) y oligoquetos (2 familias).

Los efemerópteros que aparecen son principalmente Heptagénidos y Bétidos, estos últimos también muy abundantes en los tramos medios y bajos. En cuanto a los dípteros, hay una gran variedad de familias, siendo predominantes los Qui- 
Tabla 2. Abundancia (individuos $/ \mathrm{m}^{2}$ ) de los invertebrados recogidos en el mes de Mayo de 2003. D: individuos recogidos en las derivas. *: nuevas citas para La Rioja. Abundance (individuals $/ \mathrm{m}^{2}$ ) of invertebrates collected in May 2003. D: specimens collected in drift samples. *: new records for La Rioja.

\begin{tabular}{|c|c|c|c|c|c|c|c|c|c|c|c|}
\hline Invertebrados & R-1 & R-2 & R-3 & R-4 & R-5 & Invertebrados & R-1 & $\mathbf{R}-2$ & $\mathbf{R}-3$ & R-4 & R-5 \\
\hline TRICHOPTERA & & & & & & Baetidae & & & & & \\
\hline Hydropsychidae & & & & & & Baetis spp. & 156 & 54 & 135 & 935 & 111 \\
\hline Hydropsyche instabilis & 26 & 0 & 0 & 0 & 0 & Caenidae & & & & & \\
\hline Hydropsyche lobata* & 0 & 1 & 0 & 0 & 0 & Caenis rivulorum & 9 & 0 & 1 & 2 & 19 \\
\hline Hydropsyche siltalai & 0 & 2 & 0 & 0 & 0 & Caenis gr. macrura & 0 & 3 & 0 & 0 & 11 \\
\hline Hydropsyche fontinalis* & 0 & 1 & 0 & 0 & 0 & COLEOPTERA & & & & & \\
\hline Rhyacophilidae & & & & & & Elmidae & & & & & \\
\hline Rhyacophila obliterata & 4 & 0 & 0 & 0 & 0 & Elmis spp. & 2 & 0 & 0 & 2 & 0 \\
\hline Rhyacophila martyinovi* & 2 & 0 & 0 & 0 & 0 & Esolus spp. & 76 & 6 & 1 & 0 & 0 \\
\hline Rhyacophila spp. & 0 & 0 & 0 & 0 & 2 & Limnius spp. & 6 & 0 & 0 & 0 & 0 \\
\hline Goeridae & & & & & & Oulimnius spp.* & 0 & 0 & 0 & D & 9 \\
\hline Larcasia partita & 9 & 0 & 0 & 0 & 0 & Stenelmis spp.* & 0 & 0 & 0 & 0 & D \\
\hline Brachycentridae & & & & & & Hydraenidae & & & & & \\
\hline Micrasema minimum* & 9 & 0 & 0 & 0 & 0 & Hydraena sp. $1^{*}$ & D & D & 0 & 2 & 0 \\
\hline Micrasema longulum & D & 0 & 0 & 0 & 0 & Hydraena sp. $2^{*}$ & D & 0 & 0 & 0 & 0 \\
\hline Sericostomatidae & & & & & & Haliplidae & & & & & \\
\hline Schizopelex spp. & 6 & 0 & 0 & 0 & 0 & Haliplus spp. & D & 0 & 0 & 0 & 0 \\
\hline Sericostoma spp. & 7 & 0 & 0 & 0 & 0 & Hydroscaphidae & & & & & \\
\hline Uenoidae & & & & & & Hydroscapha spp. & 0 & 1 & 0 & 0 & 0 \\
\hline Thremma spp.* & 2 & 0 & 0 & 0 & 0 & Dytiscidae & & & & & \\
\hline Psychomyidae & & & & & & Yola bicarinata* & 0 & 0 & 2 & 0 & 0 \\
\hline Psychomyia pusilla & 0 & 1 & 0 & 0 & 0 & Agabus spp. & 0 & 0 & D & 0 & 0 \\
\hline Hydroptilidae & & & & & & DIPTERA & & & & & \\
\hline Stactobia spp.* & 0 & 0 & 0 & D & 0 & Psychodidae & & & & & \\
\hline PLECOPTERA & & & & & & Psychoda spp. & 0 & 0 & 0 & 2 & 7 \\
\hline Perlidae & & & & & & Pericoma spp. & D & 0 & 1 & 2 & 0 \\
\hline Dinocras cephalotes & 22 & 1 & 0 & 0 & 0 & Blephariceridae & & & & & \\
\hline Perlodidae & & & & & & Blepharicera fasciata & 2 & 0 & 0 & 0 & 0 \\
\hline Isoperla moselyi* & 0 & 13 & 7 & 6 & 0 & Simuliidae & 2 & 10 & 6 & 183 & 33 \\
\hline Isoperla spp. & 2 & 0 & 0 & 0 & 0 & Ceratopogonidae & & & & & \\
\hline Hemimelaena flaviventris & 0 & 0 & 0 & 6 & 0 & Palpomia spp. & 0 & 1 & 6 & 0 & 2 \\
\hline Chloroperlidae & & & & & & Chironomidae & & & & & \\
\hline Siphonoperla torrentium & 7 & 0 & 1 & 0 & 0 & Tanypodinae & 30 & 31 & 61 & 11 & 4 \\
\hline Taeniopterygidae & & & & & & Chironominae & & & & & \\
\hline Rhabdiopteryx thienemanni* & 6 & 1 & 0 & 0 & 0 & Tanytarsini & 111 & 4 & 18 & 54 & 28 \\
\hline Brachyptera spp. & 0 & 40 & 44 & 10 & 11 & Chironomini & 0 & 12 & 4 & 19 & 0 \\
\hline Taeniopteryx shoenemundi* & 0 & 0 & 0 & 2 & 0 & Orthocladiinae & 83 & 104 & 761 & 2151 & 3733 \\
\hline Nemouridae & & & & & & Limoniidae & & & & & \\
\hline Protonemura spp. & 7 & 1 & D & 0 & 0 & Dicranota spp. & 4 & 1 & 0 & 5 & 0 \\
\hline Nemoura spp. & D & 0 & 1 & 2 & 0 & Antocha vitripennis & D & 0 & 0 & 0 & 0 \\
\hline Capniidae & & & & & & Stratiomyidae & 0 & 0 & 0 & 2 & 0 \\
\hline Capnia spp. & 6 & 0 & 0 & 0 & 0 & Empididae & & & & & \\
\hline EPHEMEROPTERA & & & & & & Hemerodromia spp. & 7 & 1 & 1 & 3 & 0 \\
\hline Heptageniidae & & & & & & Atalanta spp. & 0 & 0 & 0 & 5 & 0 \\
\hline Ecdyonurus forcipula/angelieri & 4 & 0 & 0 & 0 & 0 & Dolichopodidae & 0 & 0 & 0 & 2 & 0 \\
\hline Ecdyonurus lateralis & 0 & 0 & 1 & 0 & 0 & Rhagionidae & 0 & 0 & 0 & D & 0 \\
\hline Ecdyonurus spp. & 2 & 0 & 0 & 0 & 0 & Athericidae & & & & & \\
\hline Rhitrogena sp. 1 & 31 & 15 & 7 & 0 & 2 & Atherix spp. & 4 & 0 & 0 & 0 & 0 \\
\hline Rhitrogena sp. 2 & 9 & 0 & 0 & 0 & 0 & Atrichops spp. & 0 & 0 & 0 & 0 & 2 \\
\hline Epeorus torrentium/sylvicola & 2 & 0 & 0 & 0 & 0 & Tabanidae & 0 & 0 & D & 0 & 0 \\
\hline
\end{tabular}


Tabla 2 (cont.)

\begin{tabular}{|c|c|c|c|c|c|c|c|c|c|c|c|}
\hline Invertebrados & R-1 & $\mathbf{R}-2$ & $\mathbf{R}-3$ & R-4 & R-5 & Invertebrados & R-1 & R-2 & $\mathbf{R}-3$ & R-4 & R-5 \\
\hline Epeorus spp. & 0 & 1 & 0 & 0 & 0 & Syrphidae & & & & & \\
\hline Heptagenia spp. & 6 & 1 & 0 & 2 & 0 & Myiatropa spp. & 0 & 0 & D & 0 & 0 \\
\hline Ephemerellidae & & & & & & Ephydridae & & & & & \\
\hline Serratella ignita & 61 & 5 & 7 & 11 & 50 & Psilopa spp. & 0 & 0 & 1 & D & 0 \\
\hline Leptophlebiidae & & & & & & HEMIPTERA & & & & & \\
\hline Habrophlebia & 0 & 0 & D & 0 & D & Corixidae & 0 & 00 & 0 & 2 & \\
\hline S.I. & 4 & 1 & 0 & 0 & 0 & & & & & & \\
\hline COLLEMBOLA & & & & & & NEMATODA & 0 & D & 0 & 8 & 0 \\
\hline Especie 1 & D & 1 & D & 2 & 4 & TURBELLARIA & & & & & \\
\hline Especie 2 & D & 0 & 0 & 0 & D & TRICLADIDA & & & & & \\
\hline ARACHNIDA & & & & & & Planariidae & & & & & \\
\hline Actimedida & 111 & 12 & 16 & 13 & 9 & Polycelis tenuis & 4 & 0 & 0 & 0 & 0 \\
\hline BRANCHIOPODA & & & & & & HIRUDINEA & & & & & \\
\hline ANOMOPODA & & & & & & ARHYNCHOBDELLIDA & & & & & \\
\hline Eurycercidae & & & & & & Erpobdellidae & & & & & \\
\hline Alona spp. & 0 & 1 & 0 & 0 & 0 & RHYNCHOBDELLIDA & & & & & \\
\hline Chydorus spp. & 0 & D & 0 & 0 & 0 & Glossiphonidae & 0 & 0 & 4 & 0 & 0 \\
\hline OSTRACODA & 41 & 6 & 4 & 3 & 6 & OLIGOCHAETA & & & & & \\
\hline COPEPODA & 2 & 4 & 4 & 16 & 15 & OPISTHOPORA & & & & & \\
\hline MALACOSTRACA & & & & & & Lumbricidae & & & & & \\
\hline AMPHIPODA & & & & & & Eiseniella tetraedra & 0 & 1 & 3 & 0 & 6 \\
\hline Niphargidae & 0 & 26 & 2 & 0 & 76 & LUMBRICULIDA & & & & & \\
\hline Gammaridae & & & & & & Lumbriculidae & 109 & 13 & 10 & 219 & 104 \\
\hline Echinogammarus spp. & 0 & 1 & 0 & 0 & 81 & TUBIFICIDA & & & & & \\
\hline ISOPODA & & & & & & Naididae & & & & & \\
\hline Asellidae & 0 & 11 & 6 & 0 & 2 & Nais spp. & 15 & 269 & 1 & 17 & 0 \\
\hline GASTROPODA & & & & & & Specaria-Piguetiella & 0 & 3 & 0 & 0 & 0 \\
\hline BASOMMATOPHORA & & & & & & Dero spp. & 0 & 0 & 1 & 0 & 0 \\
\hline Planorbidae & 0 & 1 & 0 & 0 & 0 & & & & & & \\
\hline
\end{tabular}

\section{Abundancia y diversidad}

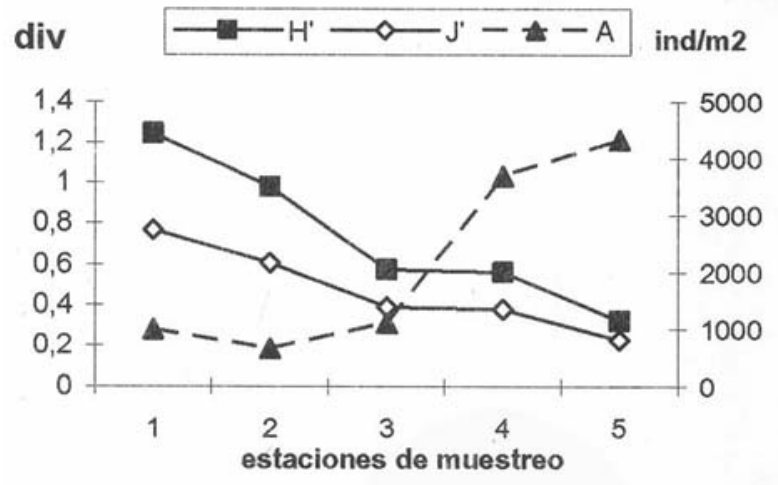

Figura 2. Abundancia total (individuos $/ \mathrm{m}^{2}$ ) y diversidad de los macroinvertebrados del río Oja (Mayo 2003). A: abundancia, $H^{\prime}$ : índice de diversidad de Shannon-Weaver, $J^{\prime}$ : índice de Pielou. Total abundance (individuals $/ \mathrm{m}^{2}$ ) and diversity of $\mathrm{ma}$ croinvertebrates of the Oja River (May 2003). A: abundance, $H^{\prime}$ : Shannon-Weaver index, $J^{\prime}$ : Pielou index. ronómidos, aunque la abundancia de las distintas subfamilias y tribus varía en los distintos puntos, p.e. los Tanytarsini (Chironominae) son mayoritarios en R-1, mientras que los Orthocladinae lo son en el resto.

Los crustáceos también tienen buena representación, con branquiópodos, ostrácodos, copépodos, isópodos y dos familias de anfípodos. En cuanto a los oligoquetos, aparecen tres familias: Lumbricidos, Lumbricúlidos y Naididos, así como dos familias de hirudíneos (Erpobdelidos y Glossifonidos).

Cuando representamos la evolución espacial de la diversidad $\left(H^{\prime}\right.$ y $\left.J^{\prime}\right)$ y la abundancia total de individuos a lo largo del río (Fig. 2), observamos que hay una disminución desde las zonas altas de la diversidad, mientras que la abundancia total aumenta, debido sobre todo a la presencia de unas pocas especies, presentes en gran número en las muestras. La abundancia más baja se sitúa en el 
punto 2 posiblemente debida a la presencia del vertido de la depuradora de Ezcaray.

\section{DISCUSIÓN}

Los resultados expuestos, aun siendo preliminares, nos muestran una fauna de macroinvertebrados muy rica, debido a la buena calidad de las aguas del río, que permitiría desarrollar las comunidades potenciales de cada tramo. Por otro lado, factores estresantes como p.e. las inundaciones del tramo medio-bajo del valle (aproximadamente un mes antes del del muestreo) y la sequía del tramo medio (puntos R-3 y R- 4), que se repite todos los años con mayor o menor intensidad (comunicación personal de habitantes de la zona), pueden ser determinantes en la distribución $\mathrm{y}$ estabilidad de las poblaciones.

En cuanto a las comunidades de macroinvertebrados, podemos decir que están compuestas por especies con distinta procedencia biogeográfica (Illies, 1978; González et al., 1992; Sánchez Ortega et al., 2002): endemismos ibéricos, como Larcasia partita (Trichoptera: Goeridae), distribuida por toda la península, Hydropsyche fontinalis (Trichoptera: Hydropsychidae), hasta ahora citada en el sur de la Península (Zamora-Muñoz et al., 2002) o Rhabdiopteryx thienemanni (Plecoptera: Taeniopterygidae). Otro grupo lo constituyen especies citadas como endémicas de Pirineos y Andorra, como Isoperla moselyi (Plecoptera: Perlodidae), Rhyacophila martynovi (Trichoptera: Rhyacophilidae) o Hydropsyche angustipennis (Trichoptera: Hydropsychidae), que aparece en las muestras de noviembre (inéditas). Hay también especies que se distribuyen por la península Ibérica y el norte de África, como $\mathrm{He}$ mimelaena flaviventris (Plecoptera: Perlodidae), o Hydropsyche lobata (Trichoptera: Hydropsychidae). En cuanto a las especies con distribución europea tenemos p.e. Dinocras cephalotes (Plecoptera: Perlidae), Hydropsyche instabilis e H. siltalai (Trichoptera: Hydropsychidae), o Rhyacophila obliterata (Trichoptera: Rhyacophilidae), algunas de distribución meridional, como Micrasema minimum (Trichoptera: Brachycentridae) y otras presentes también en el cen- tro, oeste y sur de Europa, como Siphonoperla torrentium (Plecoptera:Chloroperlidae), Taeniopteryx shoenemundi (Plecoptera: Taeniopterygidae), o Micrasema longulum (Trichoptera: Brachycentridae). Toda esta variedad de procedencias, con una mayoría de especies endémicas de la Península, confiere un gran valor ecológico a la entomofauna del río Oja, que se verá incrementado con los resultados del resto de los meses estudiados. Por este motivo, es necesario un estudio en mayor profundidad de estas comunidades dulceacuícolas, con vistas a una protección eficaz de su hábitat.

\section{AGRADECIMIENTOS}

Este trabajo de investigación se ha financiando a través del Proyecto del Plan Nacional de I+D (REN2002-02550), actualmente en curso, y cuenta con el apoyo de la Unión de Agricultores y Ganaderos-Coordinadora de Agricultores y Ganaderos (UAGR-COAG) mediante un Contrato Tecnológico (ref. 2003/672). La Consejería de Educación de la Comunidad de Madrid y el Fondo Social Europeo (F.S.E.) participan mediante la financiación de una beca de Formación de Personal Investigador.

\section{REFERENCIAS}

AUBERT, J. 1963. Les Plécoptères de la Péninsule Iberique. Eos, 39: 23-107.

BERTRAND, H. 1954. Les Insectes aquatiques d'Europe. Vols. I y II. E. Lechevalier, Paris, 2 vol., 566 p.

DESPAX, R. 1936. Contribution à l'étude du genre Chloroperla (Pictet) (Isoperla Banks). Bull. Soc. Hist. nat. Toulouse, 69: 337-398.

GARCÍA DE JALÓN, D. 1982a. Contribución a la zoogeografía de los Tricópteros españoles (I). Shilap, Rev. Lepid.,10 (37):75-78

GARCÍA DE JALÓN, D. 1982b. Contribución a la zoogeografía de los Tricópteros españoles (II). Shilap, Rev. Lepid.,10 (38): 157-165.

GARCÍA DE JALÓN, D. 1982c. Contribución a la zoogeografía de los Tricópteros españoles (II). Shilap, Rev. Lepid.,10 (39): 237-244.

GONZÁLEZ, M. A., L. S. W. TERRA, D. GARCÍA 
DE JALÓN \& S. COBO. 1992. Lista faunística y bibliográfica de los Tricópteros (Trichoptera) de la Península Ibérica e Islas Baleares. Asociación Española de Limnología, Publicación n ${ }^{0} 11$, Madrid. $200 \mathrm{pp}$.

ILLIES, J. 1978. Limnofauna Europaea. Ed. G. Fisher, Stutgart. 523 pp.

MARTÍNEZ-BASTIDA, J. J., ARAUZO, M., VALLADOLID, M. 2006. Diagnóstico de la calidad ambiental del río Oja (La Rioja, España) mediante el análisis de la comunidad de macroinvertebrados bentónicos. Limnetica, 25 (3): 733-744.

NAVÁS, L. 1914. Notas entomológicas. Excursión anual de la Sociedad Aragonesa de Ciencias Naturales a Ortigosa y Valvanera (Logroño). Bol. Soc. Arag. Cienc. Nat., 13: 29-37.

NAVÁS, L. 1917a. Tricópteros (Ins.) de España. Asoc. Esp. Progr. Cienc., Congreso de Sevilla, 5-16.

NAVÁS, L. 1917 b. Neurópteros nuevos o poco conocidos (Octava serie). Mem. R. Acad. Cienc. Art. Barcelona, 13: 155-178.

PUIG, M. A. 1980. Contribució a l'etudi de l'ecologia comparada dels Plecòpters i Efemeròpters d'Andorra. Bull. Inst. Cat. Hist. Nat., 45: 77-87.

PUIG, M. A. 1984. Efemerópteros y Plecópteros de los ríos catalanes. Tesis Doctoral, Universidad de Barcelona. 582 pp.

SÁNCHEZ ORTEGA, A., J. ALBA TERCEDOR \& J. M. TIERNO DE FIGUEROA. 2002. Lista faunística y bibliográfica de los Plecópteros (Plecoptera) de la Península Ibérica e Islas Baleares. Asociación Española de Limnología, Publicación n 16 , Madrid. 198 páginas.

TACHET, H., P. RICHOUX, M. BOURNAUD et P. USSEGLIO-POLATERA. 2003. Invertébrés d'eau douce. Systematique, biologie, écologie. CNRS Edit., Paris. 587 pp.

VALLADARES, L. F., J. A. DÍAZ y J. GARRIDO. 2000. Coleópteros acuáticos del Sistema Ibérico Septentrional (Coleoptera: Haliplidae, Gyrinidae, Dytiscidae, Hydraenidae, Helophoridae, Hydrochidae, Hydrophilidae). Boln. Asoc. Esp. Ent. 24(3-4): 59-84.

VIEIRA, R. 2000. Las larvas de los Tricópteros de Galicia (Insecta: Trichoptera). Tesis Doctoral, Universidad de Santiago de Compostela. 612 pp.

ZAMORA-MUÑOZ, C. 2002. Hydropsyche fontinalis, a new species of the instabilis-group from the Iberian Peninsula (Trichoptera, Hydropsychidae). Aquatic Insects, 24(3): 189-197. 
